\title{
System of Monitoring and Guidance of Autonomous Vehicles based on Machine Vision
}

\author{
Christian E. Jaimes M, Julio D. Garcia L and Julian R. Camargo L* \\ Engineering Faculty, Universidad Distrital Francisco Jose de Caldas, Bogota D.C., Colombia; \\ jcamargo@udistrital.edu.co, cejaimesm@correo.udistrital.edu.co, jdgarcial@correo.udistrital.edu.co
}

\begin{abstract}
Objectives: To describe the design and implementation of an autonomous vehicle monitoring and guidance system (AGV) based on machine vision. Methods/Analysis: The design of each of the modules was established: Machine vision that obtains the position, orientation and identification of each AGV from the recognition of the detector codes. The wireless communication interface from the use of the Xbee module and that establishes the communication between the AGVs and the central host. And the central controller makes the decision of the host for each AGV as the control of traffic and movement. Findings: For each of these modules the relevant tests were carried out in 3 stages in which the first one tested the HMI that allows the user to control the whole system. The second stage tests were performed on each of the modules for one and two cameras, and the last stage examines the behavior of the complete system from the data obtained from the second stage. Improvements: By using a better resolution camera the system can operate with better results.
\end{abstract}

Keywords: Detector Code, Host, Machine Vision, Wireless Communication, AGV

\section{Introduction}

An AGV (Automatic Guided Vehicle) is a vehicle for industrial use responsible for transporting supplies, materials and final products from one point (station) to another in a well-defined area of a factory or industrial environment 1 . These vehicles are automatically controlled by a centralized computer system (host) in which there is no direct human intervention. The host is in charge of the control of the dispatches made by each AGV of the products from one station to another, the traffic control of the system and the verification of the operation status of each AGV. These orders from the host to the AGVs are executed along the established route to complete a series of tasks that the AGVs must carry out over the wireless network.

The navigation of the AGVs is done in different ways. A widely used method is to guide the AGV by means of magnetic strips installed on the floor of the factory with defined trajectories. Another method uses reflectors located on some walls of the factory to guide the AGVs that have a laser beam located at the top. AGVs with optical guidance are also used, that is, using proximity sensors, which detect walls, objects or even other nearby AGVs. The drawbacks of these methods are:

- For the magnetic strip method, large amounts of time are consumed both in the installation and in the dismantling of trajectories; the installation cost increases as the trajectories are longer which makes them less flexible.

- For the laser guidance system, a laser with its respective turning system and one sensor is required for each AGV. The number of reflectors increases with the size of the trajectories that the AGVs must travel and this fact directly affects the cost. It is not very flexible because for each changed route the positions of the reflectors must be modified.

- The optical guidance method has the disadvantage of the large number of sensors used throughout the AGV system. They are also little used systems in open environments and are only efficient on narrow roads like corridors. 
It is sought to show in this paper a system that overcomes the previously described inconveniences. The idea is to use the machine vision to obtain the information of the positions of each of the AGVs through one or more digital cameras, located in fixed positions and whose fields of vision are different among them, to increase the visual coverage of the factory or work areas of the AGVs. From this information obtained, a central system is responsible for the control of trajectories, traffic management, loading and unloading, detection of vehicle failures and activity registration of the AGVs according to the scheduled transport activities.

The advantages of the method of machine vision guidance with respect to the most commonly used methods are:

- Cost Reduction: As the number of cameras used depends only on the size of the work area and not on the number of AGVs. In addition, a camera covers an area larger than the sensors of the other systems.

- Greater Flexibility: Since the programming of the activity of each AGV and the trajectories can be modified for the most part by software and do not require additional physical facilities, which allows the number of trajectories that can be programmed to be much greater with respect to the other methods, without increasing the cost.

- Greater Speed: Because the programming of the trajectories can be done without stopping the production and this change only requires software modifications.

- Greater Capacity of Monitoring of the AGV Units: This is due to the fact that the digital camera covers a much larger area than the sensors of the other systems, and thanks to the growing technologies of obtaining digital images, more information can be obtained in the same area, capturing with a single image the positions of all the AGV.

- Easier Installation: Since a less elaborate infrastructure is required than the other systems and a smaller number of personnel.

\section{Background}

The use of AGVs is important to automate the transport of products and materials within a factory or industrial environment. Since the invention of the AGV there has been a need in the improvement of its design from the control part to the guidance systems, in order to increase the transport performance of the AGV and thus obtain lower economic cost of a given company.

There are several ways to approach guided systems for AGV using machine vision; the most important ones are summarized below.

The vision system called VISOCAR 2 for the orientation of the mobile robot in industrial environments, is an optical navigation AGV (automatically guided vehicle) which, due to its local intelligence, can travel automatically in its natural environment, does not need no installation of special floors, and does not impose restrictions on the network of routes or the environment of the factory.

Another method uses a vision system based on the stereoscopic principle ${ }^{3}$, which has been applied to an automated guided vehicle system (AGV) successfully tested in Machinery Laboratory of the Jillin Technological University. Two images taken by two cameras that are placed in the same reference plane are used, this visual information is transmitted to a PC through a video interface, using an algorithm used to create the shortest route that each AGV.

The image processing ${ }^{4}$ can be used to develop a control system for the AGVs, this in order to return the AGVs to their respective guidance line when they leave it. A PLC is used for the application of AGV control. A Wireless CCD Camera is also used as a sensor for the detection of the guide line; the camera is located on the front of each AGV. The use of color CCD cameras ${ }^{\underline{5}}$ is another method for driving an AGV; in this case the image processing was used to guide the AGV to its destination by measuring the condition of the guide and the status of each AGV.

The adaptive control called Controller Mediated Immune Algorithm (CMIA) $\underline{6}$ allows AGVs to be controlled more efficiently, since the transport of AGVs improves as they adapt to the work area.

Through visual intelligence ${ }^{-}$tools can be developed to assist an AGV using neural techniques and stereovision, in this case aimed at the identification of trajectories, obstacle detection and distance measurement.

\section{Methodology}

\subsection{Machine Vision}

The machine vision ${ }^{-8}$ has the ability to detect AGVs with good accuracy thanks to the fact that the cameras are composed of pixels taking advantage of an adequate resolution (in this case it is $640 \times 480$ ) this allows an 
image contains 307200 pixels and with this the system obtains a great information of the work area from a single image. With a lower resolution makes recognition difficult because of the little information it represents in each image taken, and with a higher resolution we have more information but it requires more image processing, therefore the recognition is more delayed. As the application is real-time, information on the positions, orientations and identification of each AGV is needed in the shortest possible time for the central controller to make the correct decisions as quickly as possible.

The coverage of the image will depend on the height of the two cameras with respect to the floor and the distance between them where the AGVs will travel. If the two cameras are higher, they will cover the cameras and the more distance between them there is also more visual coverage but we must bear in mind that being at a certain distance there is an overlap between the two images whose increase implies less visual coverage.

The detector code of AGVs is based on the QR (Figure 1) codes that are useful for storing information in a dot matrix or a two-dimensional barcode. Although initially used to register spare parts in the area of vehicle manufacturing, today QR codes are used for inventory management in a wide variety of industries?

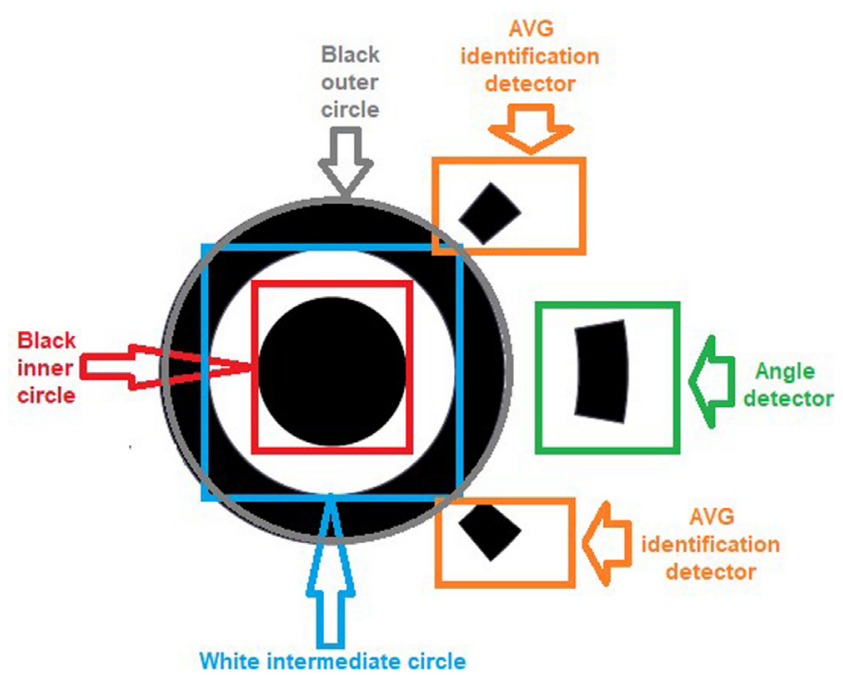

Figure 1. AGV detector code.

The code is made up of a set of circles that one is the outermost black, the intermediate one is white, and one inner that is black. All these circles are on a white background surface. All of these circles have a certain ratio that is $1: 1: 3: 1: 1$. The size of each code is $1.5 \mathrm{~cm}$ radius in the middle circle is $2.5 \mathrm{~cm}$ and the outer circle is $4.5 \mathrm{~cm}$. The most extreme part of the code is a black semicircle of $10^{\circ}$ opening that indicates the orientation of the corresponding AGV (angle indicator). The other semicircles have $10^{\circ}$ of opening and have a $90^{\circ}$ separation from each other (AGV identifier indicators). The left end corresponds to the first identification bit and the second bit corresponds to the right end. This forms the identification number of the AGV.

The first process to recognize the detector codes of each AGV is necessary the transformation of a gray scale image to a binarized image (Figure 2), according to the intensity of each pixel, with this process is done with the Otsu algorithm $\mathrm{m}^{10}$ that allows the processing of the image in bit scale generating more rapid recognition of the detector codes.

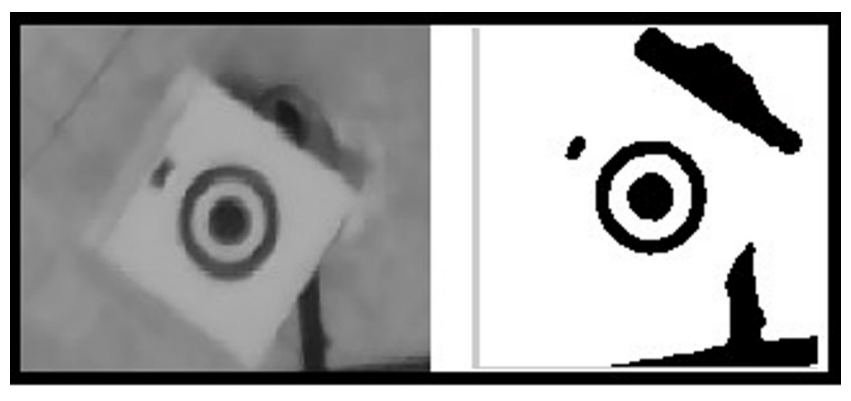

a)

b)

Figure 2. (a) Original image, (b) Binarized image.

The second process is to recognize the position of each AGV this is done by vertical and horizontal sweeps of each AGV counting the black and white stripes, and that from the proportion of the code is proceeded first confirmation of the discovery of the code from the sweep horizontal with equation (1) and then its position is determined from the vertical sweep in the center of the code using equation (2).

$$
X=- \text { cont }_{2}-\operatorname{cont}_{1}-\frac{\operatorname{cont}_{3}}{2}+m
$$

Where cont ${ }_{1}$ is the counted value of the first black band, cont ${ }_{2}$ is the counted value of the second white band, cont $_{3}$ is the value counted horizontally of the black inner circle and $m$ is the component in $X$ where the horizontal sweep was started.

$$
Y=-\frac{\operatorname{cont} V_{3}}{2}+k
$$


Where $\operatorname{cont} V_{3}$ is the value counted vertically of the inner circle black and $k$ is the component in $Y$ where the vertical sweep was started.

The third process of recognition of the detector code is to find the orientation of the AGV by calculating the distance between the found center and the angle indicator in pixels and then with a binarized image an AND logical operation is performed with the code, the result of which is a binarized image that only the angle indicator will appear.

The last process is the identification of the AGV by means of the value of the pixel value of the binarized image that is at $45^{\circ}$ of the angle indicator plus in the anti-clockwise direction which is the first bit of the AGV identifier, as well as the second bit but it is at $45^{\circ}$ more in the clockwise direction.

For the recognition of the codes with the use of the two cameras it is necessary to know that the overlap is the part formed by the intersection between the two cameras.

This overlap will depend on the height and distance between the two cameras. Overlapping is important because it makes recognition possible with two or more cameras. The minimum overlap must be equal to the width of the code in pixels that the camera is observed. It is important that the overlap be as small as possible but your area should not be zero. The minimum area of overlap should be as little as possible because if the machine vision observes an $\mathrm{AGV}$ in the area of overlap, the same detector code will be seen twice in the case of two cameras, therefore it is an information redundant and it is therefore twice the same code is processed creating more inefficiency in the recognition of the code in question (Figure 3).

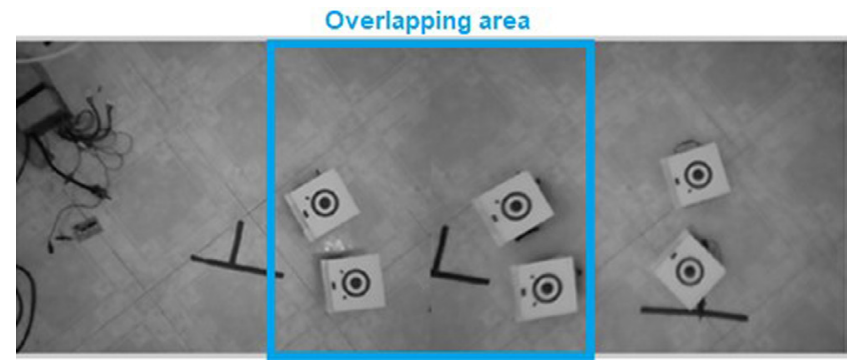

Figure 3. Overlapping area from the intersection of two images.

\subsection{General Scheme of AGV System and Central Controller}

The general scheme of the AGV system consists in the implementation of a central control in charge of controlling, monitoring and diagnosing each AGV. It is a fully centralized control, that is, all decisions from the smallest degree to the priorities of the system will be taken only by a single host or controller, this implies that the AGVs will only be responsible for receiving the host's orders, fulfilling the assigned tasks and collect some important data for the system (Figure 4).

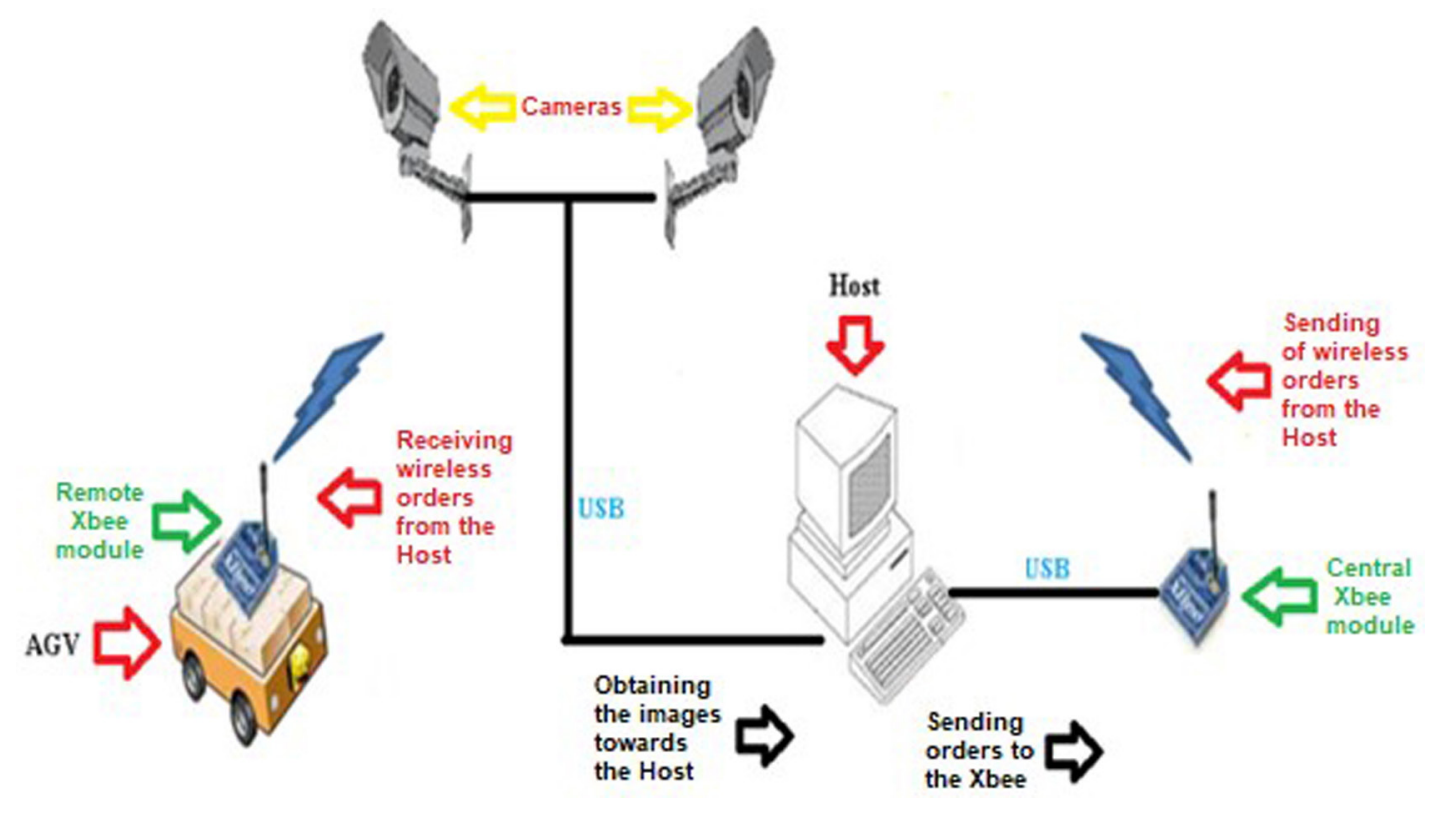

Figure 4. Pictorial diagram of the AGV system. 
The connection of the Host with the AGVs is wireless, with a configuration of star network (point to multipoint) where the transmitter is connected to the Host and the receivers are owned by the AGV.

In this scheme they also have 2 webcams; they are responsible for monitoring with machine vision to each AGV. The cameras are placed by means of one or several supports in the highest part of the work environment and have an approach that points perpendicularly to the ground, in order to have a flat and perpendicular panorama to the floor where the AGVs will be mobilized and thus allowing better observe the codes that are on top of them.
The system begins with the controller starts to execute with the initialization of global and local variables of the system in which the most important are: Position, and orientation of each AGV, the positions of the intersections of the routes and the positions of the stations.

Then the user can select three different operating modes: Simulation, maintenance and execution. If the user activates the maintenance mode, he/she can do it in the machine vision mode, in this case the systems already has a configuration by default that allows the use of the two cameras and display them in the GUI (Graphical User Interface). Or as a wireless communication that allows the diagnosis of the remote Xbee wireless system that each AGV has (Figure 5).

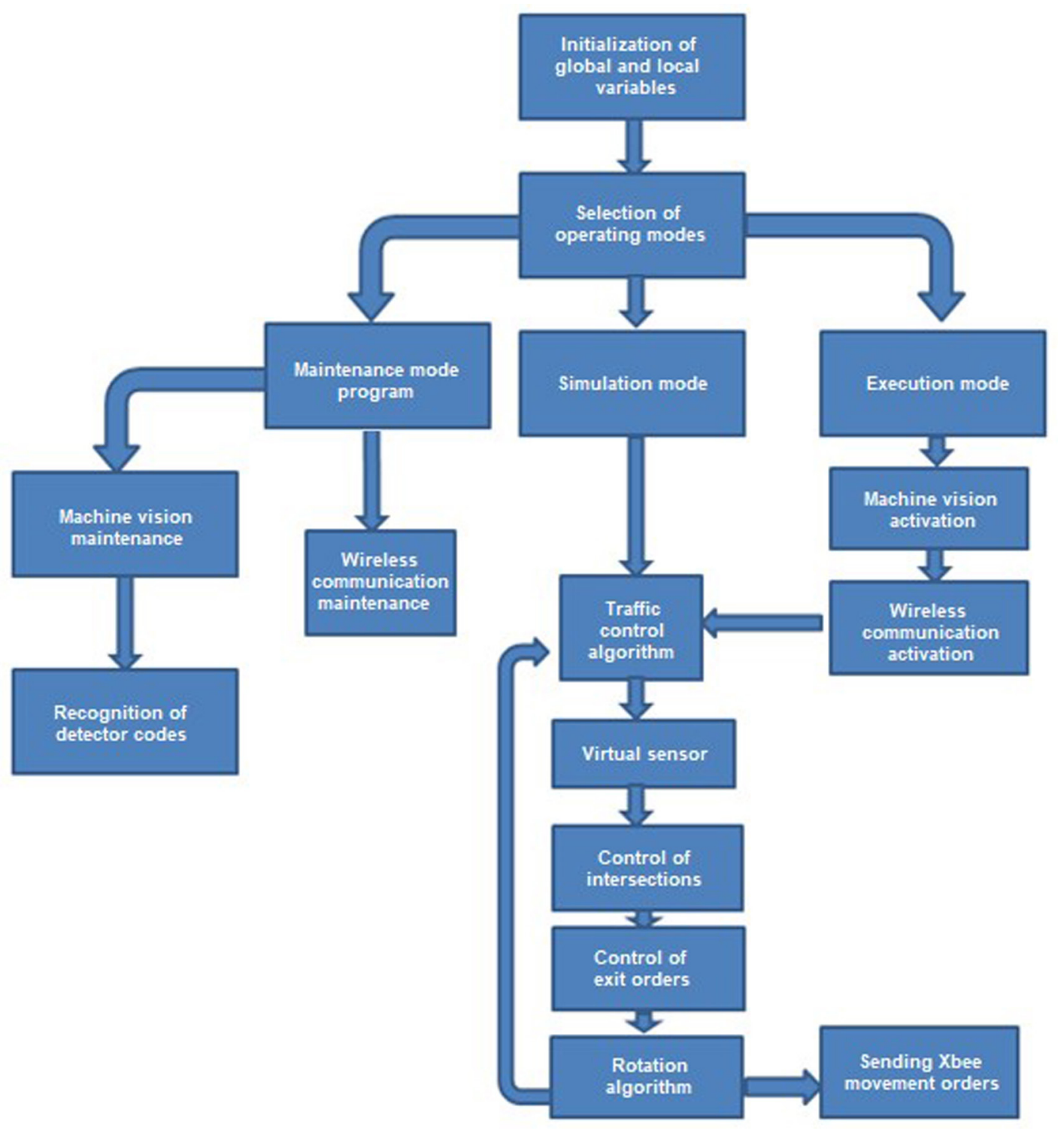

Figure 5. General scheme of the central controller. 
If one wants to work in simulation mode, that is, in this case the one will not work with real AGVs but will simulate all the actions that each AGV will perform as if the one were in a real track, in this mode it is activated several to the algorithms such as: routing algorithm that generates the shortest route that the AGV must travel between stations from the Dijkstra algorithm ${ }^{11}$; the virtual sensor that emulates and replaces a proximity sensor of an AGV and serves to detect another one that meets the forehead and has the purpose of preventing collisions between them; and the traffic control is to manage the movement of each AGV on the route and to arrive at their respective stations without any damage due to collisions.

Finally, if the active user in the execution mode activates all the algorithms described above but works with the real AGVs moving on the track, it is necessary to activate the machine vision modules and the wireless communication interface.

\section{Performance of Tests}

For the realization of tests, it was divided in three stages for a better compression of the system. The objective of these tests is to study the behavior of the AGV system; with this we provide this information for those who wish to implement this system to the users in the best possible way.
The first stage is the use of the GUI that controls the whole system by the user. The purpose of the GUI is to facilitate the understanding and control of the system by the user in a pleasant way of each AGV in real time. There is the possibility to enter the data and obtain the output data of the central controller in the MATLAB command line, but it is difficult in this way to analyze this data and can generate confusion when managing the controller (Figure 6).

The second stage is the study of each of the modules that use this AGV system, studying their behavior depending on the variation of the number of AGVs, heights and distances between cameras, among others.

The execution time of the routing algorithm will depend on the number of intersections. In this study, the execution times were collected by varying the number of intersections from 5 to 20 . Table 1 shows the results obtained, and in Figure 7 the graph that relates the data in the table is presented.

Table 1. Time record of the routing algorithm with variation of intersections

\begin{tabular}{|l|c|c|c|c|c|c|}
\hline $\begin{array}{l}\text { Number of } \\
\text { Intersections }\end{array}$ & 5 & 10 & 12 & 15 & 18 & 20 \\
\hline Time (s) & 0.0031 & 0.0057 & 0.008 & 0.0092 & 0.011 & 0.0121 \\
\hline
\end{tabular}

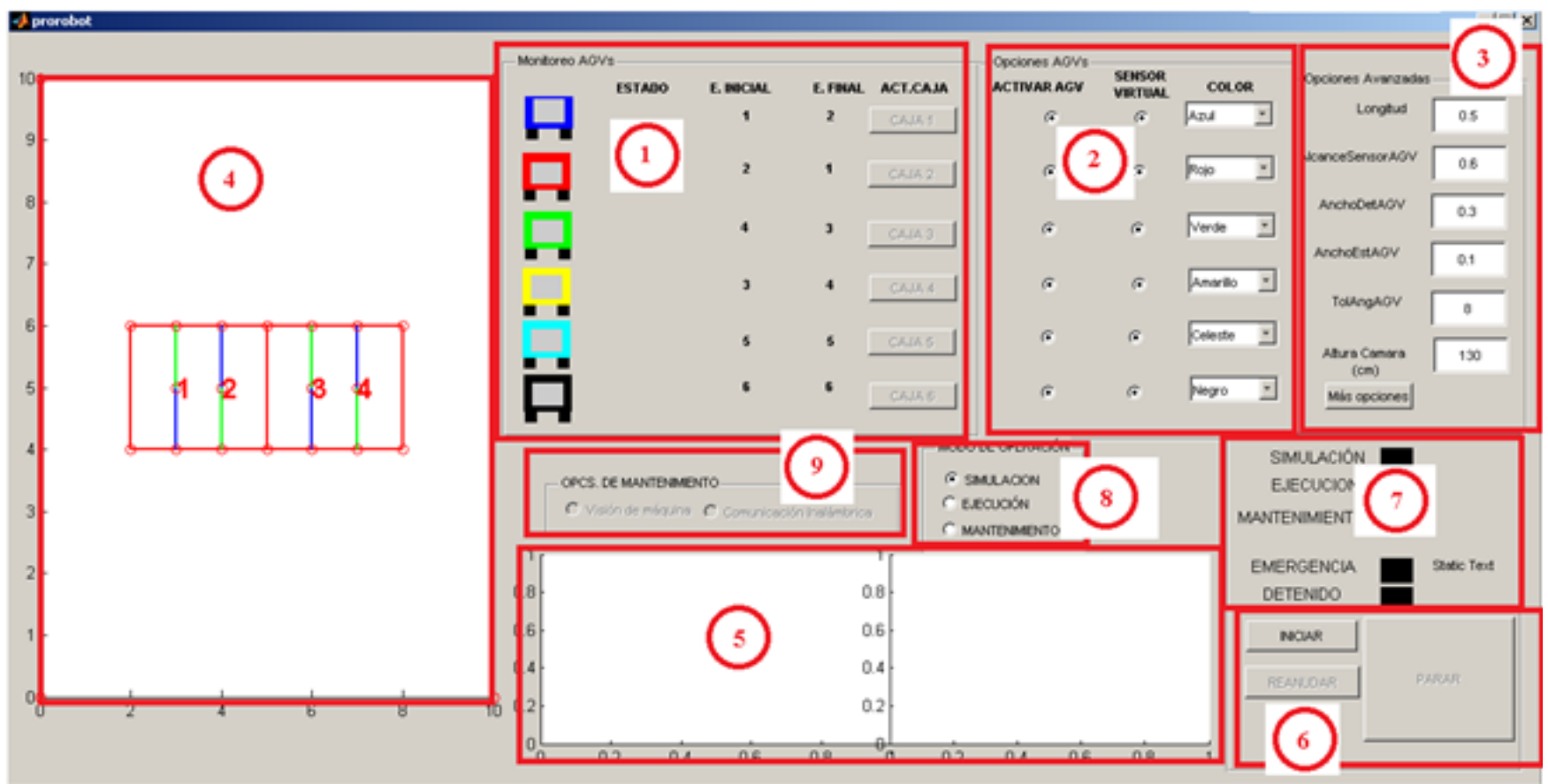

Figure 6. Conformation of the main GUI. 


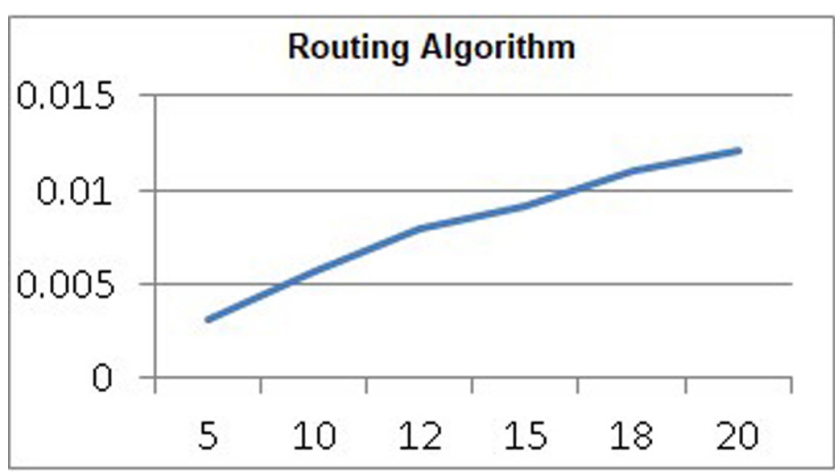

Figure 7. Time record of the routing algorithm with variation of intersections.

Table 2 recorded the execution times of the Xbee communication mode by varying the amount of AGVs activated.

Table 2. Time record of the Xbee communication module with respect to the number of activated AGVs

\begin{tabular}{|l|c|c|c|c|}
\hline Number of AVGs & 1 & 2 & 3 & 4 \\
\hline Communication time (s) & 0.1261 & 0.2837 & 0.4013 & 0.5784 \\
\hline
\end{tabular}

Tests were also carried out to recognize the detector codes for two cameras that were measured for a distance between the cameras of 1 meter, obtaining the results shown in Table 3 and in Figure 8.

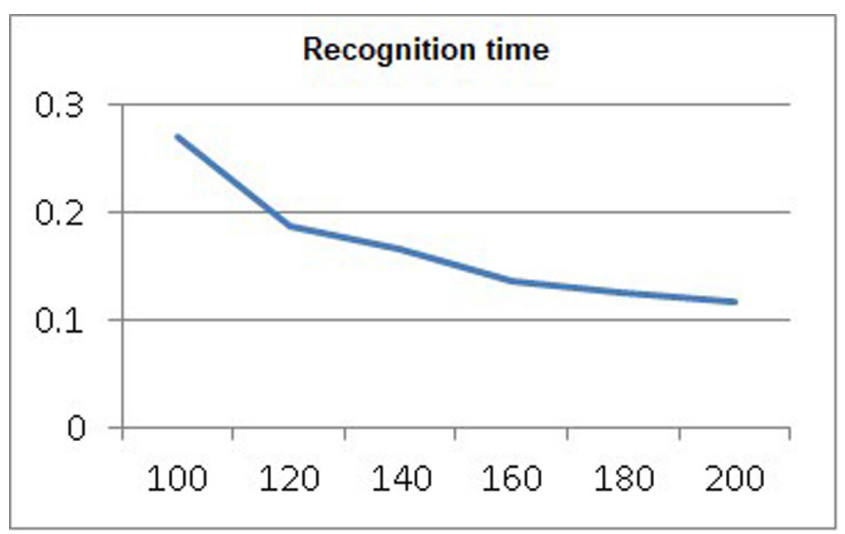

Figure 8. Record of recognition times with respect to the height of the camera.

Table 3. Analysis of overlap data and effective area of machine vision at a height of $100 \mathrm{~cm}$

\begin{tabular}{|l|c|c|c|c|c|c|}
\hline Height (cm) & 100 & 120 & 140 & 160 & 180 & 200 \\
\hline $\begin{array}{l}\text { Overlap } \\
\text { width (pix) }\end{array}$ & -19.1 & -9.7 & -2.9 & 2.1 & 6.1 & 9.3 \\
\hline $\begin{array}{l}\text { Overlap } \\
\text { width (cm) }\end{array}$ & -33.7 & -20.4 & -7.2 & 6.0 & 19.2 & 32.5 \\
\hline
\end{tabular}

\begin{tabular}{|l|c|c|c|c|c|c|}
\hline $\begin{array}{l}\text { Overlapping } \\
\text { area }\left(\mathbf{m}^{2}\right)\end{array}$ & -0.2 & -0.1 & -.06 & 0.05 & 0.2 & 0.4 \\
\hline $\begin{array}{l}\text { Effective work } \\
\text { width }(\mathbf{c m})\end{array}$ & 151.7 & 168.8 & 188.5 & 209.9 & 232.4 & 255.7 \\
\hline $\begin{array}{l}\text { Effective work } \\
\text { area }\left(\mathbf{m}^{2}\right)\end{array}$ & 0.9 & 1.2 & 1.6 & 2.0 & 2.5 & 3.16 \\
\hline
\end{tabular}

The last stage was carried out in execution mode, where the measurements of the complete system were recorded from the data obtained from the second stage. The times shown in Table 4 are in percentages of the total time necessary to execute all the tasks in the system. Presents these results graphically.

Table 4. Percentages analysis of complete system times varying the amount of AGVs

\begin{tabular}{|l|c|c|c|c|}
\hline Amount AVGs & 1 & 2 & 3 & 4 \\
\hline Communication percentage & $52 \%$ & $54 \%$ & $52 \%$ & $54 \%$ \\
\hline Traffic percentage & $6 \%$ & $4 \%$ & $3 \%$ & $3 \%$ \\
\hline Machine vision percentage & $42 \%$ & $42 \%$ & $45 \%$ & $44 \%$ \\
\hline Total Percentage & $100 \%$ & $100 \%$ & $100 \%$ & $100 \%$ \\
\hline
\end{tabular}

It can be seen that the time that is used more is by wireless communication, but the tendency of the machine vision is growing as the number of AGVs increases. The percentage of traffic time decreases as the amount of AGV increases, which indicates that traffic time is becoming less predominant and the rest of the systems (machine vision and wireless module) are the ones that will dominate.

\section{Conclusions}

It is possible to manage the guidance and traffic of AGVs through a centralized control. The system is flexible in that you can create routes from a secondary GUI that allows you to program the assignments of stations and routes only by software.

The machine vision guidance system will only depend on the cameras and no additional infrastructure is required. With this guidance system it is possible to save components such as proximity sensors or shock detectors.

In the simulation mode the user was allowed to study the behavior of the AGVs emulating the route in the work area and make the modifications of the routes in a virtual way to avoid problems in traffic such as a traffic jam or collisions between AGVs and observe traffic of vehicles 
allowing a change of parameters in traffic management to improve it and avoid these problems before actually implemented.

In the maintenance mode, the whole system was diagnosed independently, it facilitated the calibration of each of the components to solve system problems in a timely manner without the AGV suffering considerable damage. The speed of machine vision recognition depends on the number of AGVs and the height of the cameras.

\section{References}

1. Shengfang L, Xingzhe H. Research on the AGV Based Robot System Used in Substation Inspection. International Conference Power System Technology. 2006; p. 1-4. https://doi.org/10.1109/ICPST.2006.321495.

2. Frohn H, Seelen W. VISOCAR: An Autonomous Industrial Transport Vehicle Guided by Visual Navigation. Proceedings, International Conference on Robotics and Automation. 1989; p. 1155-9.

3. Feng Y, Hong C, Lanjun Z. Path Plan Acquisition System for AGV with Dual CCD-Cameras. Proceedings of the IEEE International Conference on Industrial Technology (ICIT'96). 1996; p. 867-9. https://doi.org/10.1109/ICIT.1996.601723.

4. Butdee S, Suebsomran A. Automatic guided vehicle control by vision system. IEEM-IEEE International Conference on Industrial Engineering and Engineering Management. 2009; p. 694-7. https://doi.org/10.1109/IEEM.2009.5373238

5. Lee J-W, Choi S-U, Lee C, Lee Y, Lee K. A study for AGV steering control and identification using vision system. ISIE-IEEE International Symposium on Industrial Electronics Proceedings. 2001; 3:1575-8.

6. Lee Y, Suh J, Lee K. Auto steering control of unmanned container transport (UCT) with vision system and mediated immune algorithm controller. 30th Annual Conference of IEEE Industrial Electronics Society, IECON. 2004; 3:2987-91.

7. Modesti M, Mateus S. Sistemas de guiado para colaboracion en navegacion de vehiculos autonomos (AGV). $6^{\circ}$ Encuentro de Investigadores Nacionales e Internacionales, Politecnico Colombiano Jaime Isaza Cadavid. 2008; p. 1-6.

8. Shen Y, Zhang Y, Yuan J. Design and Implementation on Motion Control System of AGV Based on DSP. IEEE 3rd International Conference on Communication Software and Networks. 2011; p. 338-42.

9. QR code.com. History of QR Code. Available from: http://www.qrcode.com/en/history/. Date accessed: 1990.

10. Otsu N. A threshold selection method from gray-level histograms. IEEE Transactions on Systems, Man, and Cybernetics. 1979; 9(1):62-6. https://doi.org/10.1109/TSMC.1979.4310076.

11. Shaikh A, Dhale A. AGV routing using Dijkstra's Algorithm - A review. International Journal of Scientific \& Engineering Research. 2013; 4(7):1665-70. 